\title{
INTERFAGETTECNOLÓGGCA
}

\section{A IMPORTÂNCIA DA TECNOLOGIA SEM FIO NA INDÚSTRIA 4.0}

\section{THE IMPORTANCE OF WIRELESS TECHNOLOGY IN INDUSTRY 4.0}

\author{
Diego Rafael Guedes dos Santos - diegogdes@gmail.com \\ Carlos Rodrigo Volante - carlos.volante@ fatectq.edu.br \\ Faculdade de Tecnologia de Taquaritinga (FATEC) - SP - Brasil
}

DOI: 10.31510/infa.v15i2.487

\section{RESUMO}

Este trabalho tem como objetivo demonstrar a importância e impactos que as tecnologias sem fio têm nas indústrias, além de acrescentar um breve conhecimento da evolução das etapas em que participaram as indústrias conforme os anos até chegarem à era da indústria 4.0, dando ênfase no estudo sobre a atuação da comunicação sem fio e quais são as principais conectividades empregadas dentro da indústria. As pesquisas bibliográficas foram elaboradas de acordo com artigos, livros e portais onde cada qual foi pesquisado minuciosamente a fonte e a veracidade de suas informações. Por fim concluiu-se que apesar de serem imprescindíveis, as tecnologias sem fio ainda têm que evoluir.

Palavras-chave: Indústria 4.0. Tecnologia sem fio. Conectividade. Revolução Industrial

\begin{abstract}
In this paper, the objective is to demonstrate the importance and impact of wireless technologies on industries, as well as give a brief summary of the evolution of the stages in which industries took part throughout the years to reach the industrial age 4.0, emphasizing the study on the performance of wireless communication and what the main connectivities employed within the industry are. The bibliographical research was elaborated according to articles, books and portals, and the accuracy of these sources was checked thoroughly. Finally, this article has shown that, although wireless technologies still have to evolve, they are an essential tool to get information.
\end{abstract}

Keywords: Industry 4.0. Wireless technology. Connectivity. Industrial Revolution 


\section{INTRODUÇÃO}

De acordo com Wang (2016), recentemente, as tecnologias emergentes, como por exemplo, internet das coisas, redes e sensores sem fio, big data, computação em nuvem, sistema embarcado e internet móvel, estão sendo introduzidas nos ambientes de produção, dando início a uma quarta revolução industrial.

Para Saltiél (2017), a indústria 4.0 ou internet das coisas, por meio da tecnologia de rede sem fio, conecta todas as partes do processo em tempo real. Máquinas, dispositivos, produtos e pessoas contribuem para uma transparência de informação única. Isso possibilita que as máquinas se comuniquem de forma autônoma, definindo tarefas e funções a serem desempenhadas para o correto funcionamento da fábrica.

Os produtos inteligentes, por meio da tecnologia sem fio, detêm as informações necessárias para o seu processo produtivo. A conexão entre a fábrica inteligente e o produto inteligente é o destaque na indústria 4.0. Os meios de produção recebem as informações por meio de diversos sensores espalhados pela fábrica, todos conectados, possibilitando a autonomia de decisão, com a própria fábrica identificando e corrigindo problemas encontrados durante o processo produtivo.

\section{A INDÚSTRIA 4.0}

A indústria 4.0 tem como conhecimento seus sistemas inteligentes, com alta capacidade de atribuir decisões autônomas. Seu crescimento inicia-se com o aumento de investimentos em áreas tecnológicas para melhorar a eficiência nos processos produtivos.

Para José Rizzo (2016), a indústria 4.0 é a descentralização dos processos produtivos e uma grande manifestação de dispositivos inteligentes interconectados dentro de uma cadeia produtiva. Rizzo ainda elucida que as indústrias estão adotando novas tecnologias voltadas à Tecnologia da Informação e incrementando na Automação Industrial, formando um sistema físico-cibernético. Com o crescimento da indústria 4.0 a informação sofre uma grande digitalização, tendo como consequência uma manufatura flexível e ajustável ao mercado.

De acordo com Bernard Marr (2016), países emergentes como a Índia poderiam se beneficiar com a indústria 4.0 aproveitando o modelo da cidade de Cincinnati, no estado de Ohio, que, por meio de relatórios, recebeu o título de "cidade de demonstração da Indústria 
4.0”. Marr acrescenta que para atingir os pilares de uma indústria 4.0 é necessário atender aos seguintes requisitos:

- Interoperabilidade

- Transparência das informações

- Assistência técnica

- Tomada de decisão descentralizada

\subsection{Revoluções industriais}

De acordo com Wright (2018), o período entre 1760 e 1840 representa a transição de artesãos qualificados que produziam sua própria mercadoria para mão de obra não qualificada, usando máquinas movidas por uma roda d'água ou motor a vapor. A transição foi mais prevalente na indústria têxtil, mas os efeitos da primeira Revolução Industrial acabaram sendo sentidos em quase todos os aspectos da vida cotidiana.

Figura 1 - Obras de máquinas em Chemnitz por volta de 1868.

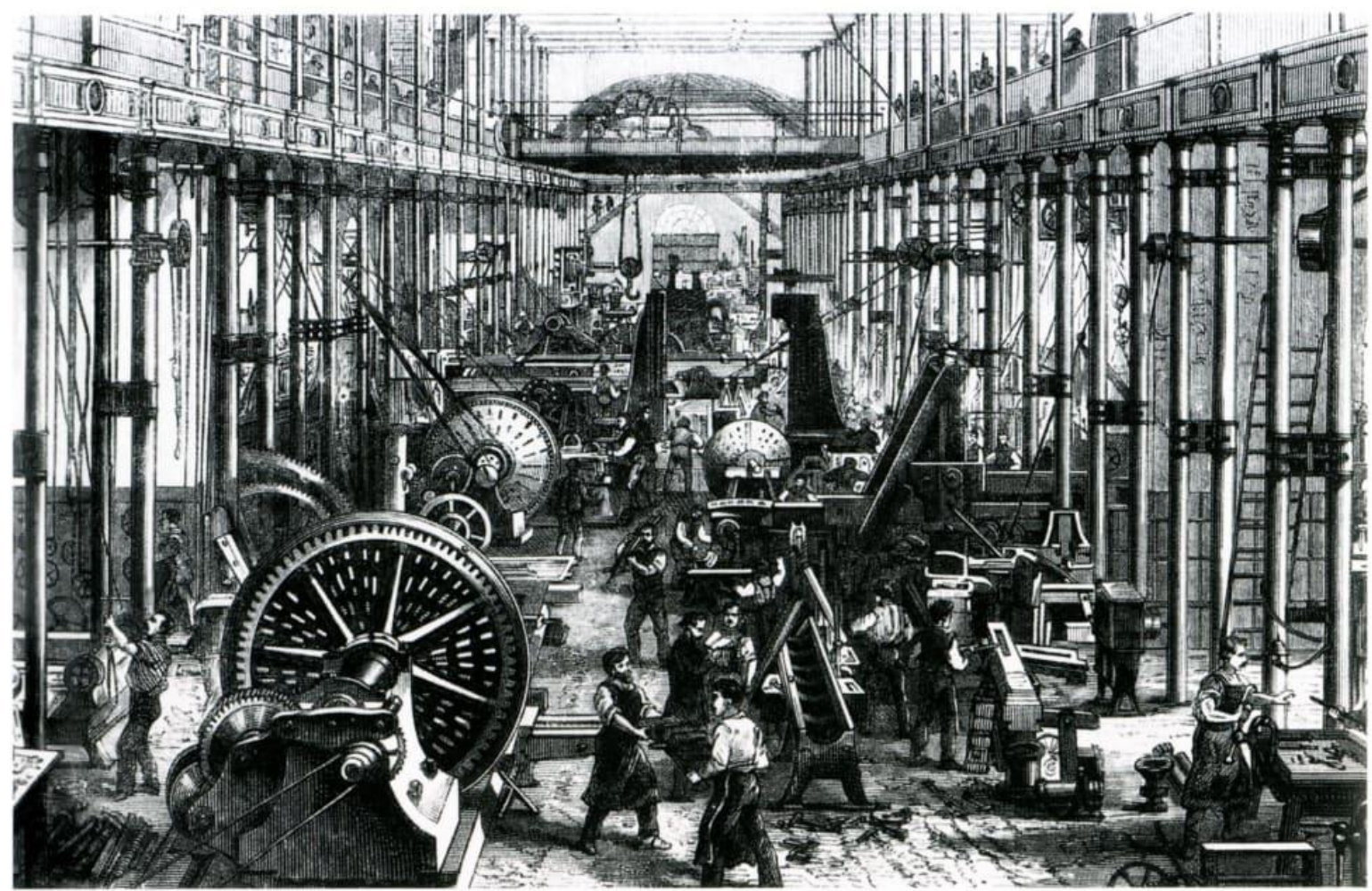

Fonte: Wright (2018) 


\section{INTERFACETECNOLÓGICA}

A segunda Revolução Industrial ocorreu durante o final do século XIX e no início do século XX, cerca de 1870 a 1914. Ao contrário da primeira Revolução Industrial, que foi caracterizada pelo advento de novas tecnologias, a segunda Revolução Industrial teve mais a ver com a melhoria das tecnologias existentes e as sinergias entre elas (WRIGHT, 2018).

O exemplo que Wright (2018) cita é a eletricidade que substituiu a água e o vapor como a principal fonte de energia nas fábricas. A Segunda Revolução industrial também marcou o início da linha de montagem, peças intercambiáveis e com elas a produção em massa.

A terceira Revolução Industrial, assim como a primeira, viu a introdução de novas tecnologias, como a automação e o computador. Esses avanços trouxeram mudanças monumentais na fabricação, permitindo níveis de precisão graças a robôs industriais e controles numéricos de computador ( $\mathrm{CNCs}$ ), nunca antes vistos no chão de fábrica. Identificar o período de tempo da Terceira Revolução Industrial é uma tarefa complexa porque, segundo alguns estudiosos, ela ainda está acontecendo, mas o começo pode ser traçado até o início dos anos 1960, que viu a introdução do primeiro robô industrial e dos primeiros CNCs comerciais (WRIGHT, 2018).

Tabela 1 - As revoluções Industriais

\begin{tabular}{ccc}
\hline $\begin{array}{c}\text { Revolução } \\
\text { Industrial }\end{array}$ & Período & $\begin{array}{c}\text { Aspectos } \\
\text { Fundamentais }\end{array}$ \\
\hline 1 & $1760-1840$ & Mecanização \\
2 & $1870-1914$ & Produção em Massa \\
3 & $1960-2000$ & Informatização \\
4 & Atualmente & Fábricas Inteligentes \\
\hline
\end{tabular}

Se tivermos uma visão ampla das últimas três revoluções industriais, surge um padrão. As revoluções ímpares foram o resultado aparente de novas tecnologias, como, o motor a vapor ou o computador. Em contraste, a revolução 2.0 teve menos a ver com a invenção de novas tecnologias do que com o aumento da sinergia entre elas (WRIGHT, 2018). 


\section{INTERFACETECNOLÓGICA}

De acordo com o ABDI (2018), as revoluções industriais anteriores tiveram uma notória contribuição para a produção em massa, linhas de montagem, eletricidade e a tecnologia da informação, conseguindo entreter a competitividade tecnológica dos meios produtivos, além do desenvolvimento econômico. A quarta revolução industrial tem grande influência na fusão de tecnologias do mundo físico, biológico e digital.

As ferramentas utilizadas pela indústria 4.0 tiveram como consequência a modificação no ambiente fabril. Dentre eles estão a produtividade, redução dos custos, controle sobre os processos produtivos e a personalização da produção (ABDI, 2018).

\section{A IMPORTANCIA DA CONECTIVDADE SEM FIO DENTRO DA INDÚSTRIA 4.0}

A conectividade sem fio traz grande flexibilidade para a empresa, além de conectar diferentes tipos de componentes em uma mesma rede, melhorando a comunicação e prosperando uma atualização de dados em tempo real.

Segundo Santos (2018), a maior parte da conectividade dentro da indústria ainda se faz por meio de redes físicas, de ponto a ponto; porém, em 2017, a conectividade dentro das indústrias já corresponde a um quarto de toda a conectividade. Podemos esperar por investimentos em longo prazo em que a indústria 4.0 revolucionará a maneira da comunicação sem fio.

De acordo com Lins (2015), a conectividade dentro das indústrias passa por várias camadas, onde essa percepção pode ser dada por vários dispositivos diferentes, contendo suas próprias características de coletar informações. Tais dispositivos podem ter conectividades como wireless, RFID, rádio frequência, Bluetooth, NFC.

A análise de Lins inclui uma arquitetura para se ter uma comunicação eficiente da fábrica ou de máquina a máquina. Essa arquitetura fundamenta-se nos seguintes itens:

- Máquinas Inteligentes: inclui comunicação máquina a máquina ou a comunicação de máquina com outros dispositivos.

- Dispositivos inteligentes: diferentes dispositivos dentro do ambiente fabril ou fora se comunicando; os dispositivos podem ser de campo, móveis ou operacionais.

- Processos de fábrica inteligentes: A comunicação dinâmica, eficiente, automatizada e em tempo real nos processos. 


\section{WWTERFAGETTECNOLÓGICA}

- Engenharia Inteligente: A percepção de melhorar os processos de acordo com dados levantados dentro e fora da fábrica.

- Fabricação de TI: Aplicações voltadas ao melhoramento e coleta de dados na indústria. Esses softwares podem ser utilizados em sensores, redes sem fio ou em qualquer tipo de aplicação de coleta da fábrica.

- Logística inteligente: incluem ferramentas para gerir o processo, além de serem uma logística autogerenciáveis, em que esta reage de modo inteligente e tem uma percepção rápida a mudanças inesperadas da produção.

- Big Data: Conjunto de dados maior e mais complexo, especialmente de novas fontes de dados. São dados armazenados em volumes crescentes e com velocidade cada vez maior.

- Fornecedores inteligentes: O compartilhamento e conectividade de informações em tempo real com o fornecedor.

\section{Figura 2 - Conectividade Wireless}

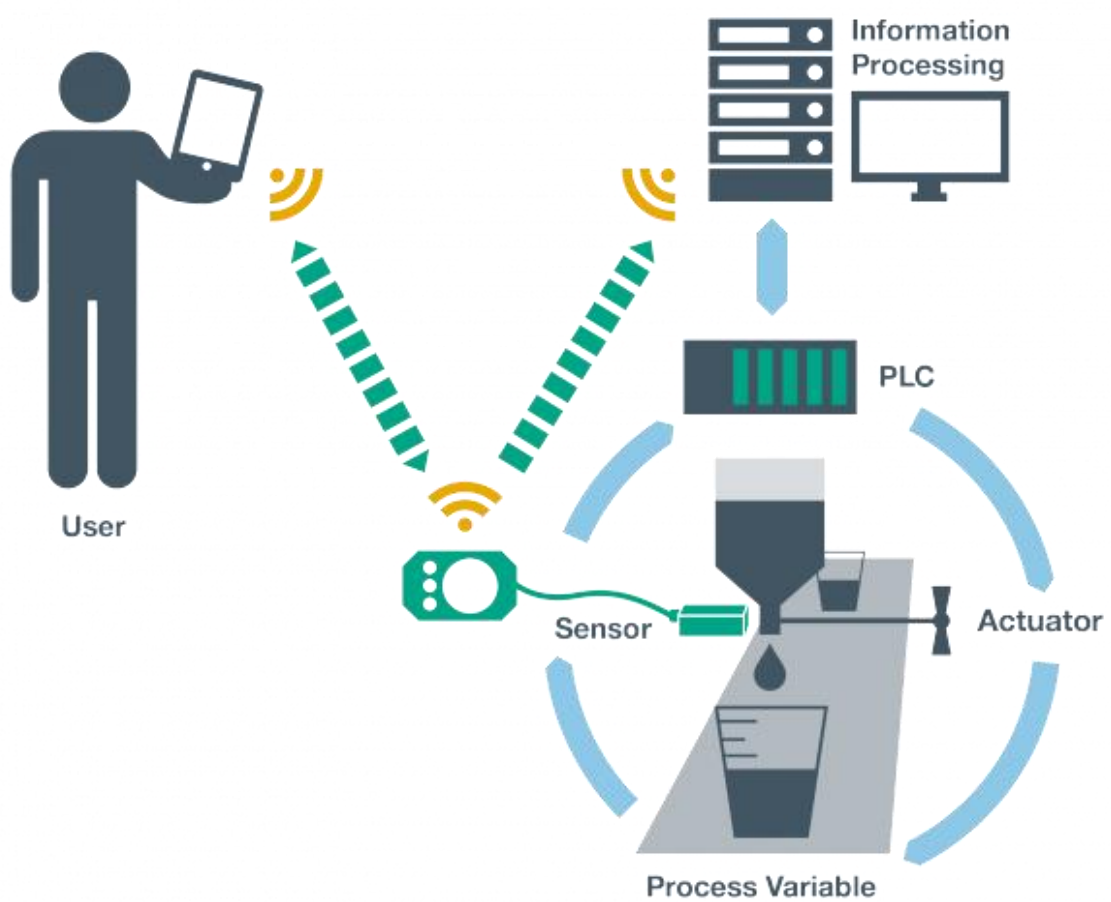

Fonte: Lins (2015) 


\section{INTERFAGE TECNOLÓGICA}

\section{TIPOS DE CONECTIVDADES SEM FIO EMPREGADAS NA INDÚSTRIA}

A indústria 4.0 emprega inúmeros tipos de dispositivos de diferentes modelos de tecnologias, usando protocolos diferentes e a criptografia peculiar de cada ambiente fabril. Apesar da peculiaridade de cada dispositivo do modo de captar as informações, a finalidade destes aparelhos é de coletar ou transmitir dados em tempo real, melhorando a eficiência de modo geral dentro da indústria.

\subsection{WiFi}

O protocolo Wi-Fi tem se destacado muito na indústria, sendo utilizado em diversas áreas. A conectividade pode ser empregada por computadores, máquinas, tablets, coletores, entre outros diversos equipamentos. Este tipo de conectividade opera em faixas de frequências de $2.4 \mathrm{Ghz}$ ou 5Ghz. Não há necessidade de licenças para a instalação na operação empregada, podendo-se obter uma velocidade de transmissão de dados de no mínimo 11 Mpbs dentro de um raio de 30 metros (LUGLI, 2012).

Segundo o site TP-Link (2013), a diferença entre as frequências é que a 2.4Ghz tem uma transmissão de dados superior, porém existem vários equipamentos que trabalham na mesma frequência resultando interferência. Já a transmissão $5 \mathrm{Ghz}$ tem um alcance menor de transmissão de dados, no entanto, devido à baixa quantidade de equipamentos que trabalham nessa mesma faixa, a transmissão tem um aproveitamento melhor. A avaliação de qual frequência usar terá como resultado a quantidade de equipamentos no local.

Esse tipo de transmissão de dados é extremamente atrativo para o ambiente industrial, conseguindo uma flexibilidade notória, porém sua transmissão de dados ainda é inferior, se comparada às redes cabeadas.

De acordo com Lugli (2012), os padrões de validação desse tipo de conectividade devem ser totalmente avaliados nos meios produtivos antes de sua implantação, para evitar erros ou perdas de dados possíveis pela arquitetura do ambiente fabril. Deve-se atentar ao local onde será implantado e onde os equipamentos vão permanecer, para evitar problemas de interferência e exposição à poeira, pó, calor e umidade. 


\section{INTERFAGETTECNOLÓGGCA}

Mesmo com as facilidades da rede WiFi, para Lugli, ela ainda não substitui as redes físicas, que servem para trabalhar em conjunto para se ter uma transmissão de dados eficiente e melhorar a mobilidade dentro do ambiente fabril.

\subsection{Bluetooth}

Apesar de ser uma tecnologia desenvolvida no inicio dos anos 90, esse tipo de conectividade só foi lançada para uso industrial no final de 1999, no inicio da indústria 4.0. Segundo Siqueira (2006), o Bluetooth é uma conexão de tecnologia via rádio frequência de baixo alcance, que serve para a transmissão de dados e troca de informações com demais dispositivos na mesma rede. Além de ser um investimento de baixo custo, o Bluetooth também consome pouca energia.

De acordo com Siqueira (2006), com uma rede baseada na arquitetura Bluetooth, pode-se obter até 8 dispositivos comunicando entre si, em um alcance de no máximo 100 metros. Podem ser utilizados em diferentes aplicações e dispositivos localizados próximos um do outro.

\subsection{RFID}

De acordo com a Totvs (2017), à medida que as empresas vão se adequando a indústrias 4.0, elas enxergam uma real necessidade de empregar a tecnologia RFID (Radiofrequency identification).

Segundo o site da empresa Totvs (2017), os RFIDs são sensores ou etiquetas que possuem microchips capazes de transmitir ou gravar dados. Essa tecnologia sem fio é empregada principalmente em sistemas de identificação ou rastreamentos de objetos e em walkie-talkies, porém ao invés de transmitir sons, emite sinais de dados.

O portal GS1 Brasil (2017), que faz parte da Associação Brasileira de Automação, acredita que a tecnologia RFID tem dentro da indústria 4.0 uma revolução no quesito de identificação de produtos nas indústrias. Tem forte impacto nos sistemas logísticos e na cadeia de abastecimento de uma forma geral. 


\section{CONCLUSÕES}

É inevitável que as indústrias de diferentes portes se adequem aos seus sistemas produtivos, ou pelo menos a uma parte deles, a introdução de novas tecnologias, como consequência, consegue ter volumes de dados onde antes era praticamente imperceptível, gerando tomadas de decisões e integrando diferentes tipos de processos, além de ter um controle geral de toda a percepção do ambiente fabril. Esse é o objetivo da quarta Revolução Industrial: aperfeiçoar processos de modo transparente do início ao fim.

A qualidade da conectividade é de extrema importância para integrar os processos de uma indústria, como cada qual tem por si sua finalidade e se destacam em diferentes tipos de ambiente.

A tecnologia que vem se destacando muito é a sem fio, por sua flexibilidade e custo, entretanto mesmo com suas vantagens ainda precisam evoluir tendo como foco auxiliar as conexões físicas dentro da fábrica.

Neste sentido, além de se apresentarem como auxiliadoras de processos, as tecnologias sem fio são também as que mais se destacam dentro da indústria 4.0, onde podemos esperar grandes evoluções da tecnologia em médio ou longo prazo.

\section{REFERÊNCIAS}

ABDI. Agenda brasileira para a Indústria 4.0.2018. Disponível em: <http://www.industria40.gov.br/>. Acesso em: 03 ago 2018.

BRUNO, Flávio da Silveira. A Quarta Revolução Industrial do Setor Têxtil e de Confecção: A visão de Futuro para 2030. São Paulo: Schäffer Editorial, 2016.

GS1. EPC/RFID e a Indústria 4.0. 2017. Disponível em: <https://www.gs1br.org/educacaoe-pratica/eventos/camp-03623-19y5s9>. Acesso em: 26 set 2018.

LINS, Theo. Industria 4.0 E IoT: Internet Das Coisas E Automação. 2015. Disponível em: <http://www.decom.ufop.br/imobilis/industria-4-0-e-iot/>. Acesso em: 20 ago 2018.

LUGLI, Alexandre Baratella. Tecnologias Wireless para Automação Industrial. 2012. Disponível em: <https://www.inatel.br/biblioteca/artigos-cientificos/2012/6088-tecnologiaswireless-para-automacao-industrial-wireless-hart-bluetooth-wisa-wi-fi-zigbee-e>. Acesso em: 26 ago 2018 
MARR, Bernard. What Everyone Must Know About Industry 4.0. 2016. Disponível em: $<$ https://www.forbes.com/sites/bernardmarr/2016/06/20/what-everyone-must-know-aboutindustry-4-0/\#aba88b0795f7>. Acesso em: 02 set 2018

ORACLE. The Definition of Big Data. 2016. Disponível em: <https://www.oracle.com/bigdata/guide/what-is-big-data.html>. Acesso em: 26 set 2018

RIZZO, José. Saiba o que é a Indústria 4.0 e descubra as oportunidades que ela gera. 2016. Disponível em: <http://www.sebrae.com.br/sites/PortalSebrae/artigos/saiba-oque-e-a-industria-40-e-descubra-as-oportunidades-que-ela-

gera,11e01bc9c86f8510VgnVCM1000004c00210aRCRD>. Acesso em: 10 ago 2018.

SAlTiÉL, Renan Mathias Ferreira. Indústria 4.0 e Sistema Hyundai de Produção: suas interações diferenças. 2017. Disponível em: <https://www.researchgate.net/publication/317369702_Industria_40_e_Sistema_Hyundai_de _Producao_suas_interacoes_e_diferencas>. Acesso em: 07 jul 2018.

SANTOS, Sandro. Introdução à Industria 4.0: Saiba tudo sobre a revolução das maquinas. São Paulo: Copyright, 2018.2 Disponível em: <https://books.google.com.br/books?id=FSRhDwAAQBAJ\&pg=PA69\&lpg=PA69\&dq=cone ctividade+sem+fio+dentro+da+industria+4.0\&source=bl\&ots=DaOCg9VDk1\&sig=qImU4er PyJEqfjJeMRRmSS9xse4\&hl=en\&sa=X\&ved=2ahUKEwj7ltjgiejdAhVIEJAKHa4FD5M4F BDoATACegQICBAB\#v=onepage $\& \mathrm{q}=$ conectividade $\% 20 \mathrm{sem} \% 20 \mathrm{fio} \% 20 \mathrm{dentro} \% 20 \mathrm{da} \% 20 \mathrm{in}$ dustria\%204.0\&f=false>. Acesso em: 15 ago 2018.

SIQUEIRA, Thiago Senador de. Bluetooth - Características, protocolos e funcionamento. $2006 . \quad$ Disponível em: <http://www.ic.unicamp.br/ ducatte/mo401/1s2006/T2/057642-T.pdf>. Acesso em: 10 set 2018.

TOTVS. A indústria inteligente exige tecnologia RFID. 2017. Disponível em: <https://www.totvs.com/biblioteca/noticias/a-industria-inteligente-exige-tecnologia-rfid>. Acesso em: 15 set 2018.

TP-Link. The differences between 2.4GHz and 5GHz Wireless. 2013. Disponível em: <https://www.tp-link.com/us/faq-499.html>. Acesso em: 01 set 2018.

WANG, Shiyong et al. Implementing Smart Factory of Industrie 4.0: An Outlook. 2016. Disponível em: <http://journals.sagepub.com/doi/full/10.1155/2016/3159805>. Acesso em: 04 mar 2018.

WRIGHT, Ian. What Is Industry 4.0, Anyway? 2018. Disponível em: $<$ https://www.engineering.com/AdvancedManufacturing/ArticleID/16521/What-Is-Industry40-Anyway.aspx>. Acesso em: 07 jul 2018. 